\title{
Multiobjective Optimization Approach for Robust Bridge Damage Identification against Sensor Noise
}

\author{
Seung-Yong Ok $\mathbb{D}^{1}{ }^{1}$ Sungmoon Jung, ${ }^{2}$ and Junho Song ${ }^{3}$ \\ ${ }^{1}$ Associate Professor, Department of Civil, Safety and Environmental Engineering, Hankyong National University, \\ 327 Chungang-ro, Anseong-si, Kyonggi-do 17579, Republic of Korea \\ ${ }^{2}$ Associate Professor, Department of Civil and Environmental Engineering, FAMU-FSU College of Engineering, \\ 2525 Pottsdamer Street, Tallahassee, FL 32310, USA \\ ${ }^{3}$ Professor, Department of Civil and Environmental Engineering, Seoul National University, 1 Gwanak-ro, Gwanak-gu, \\ Seoul 08826, Republic of Korea \\ Correspondence should be addressed to Seung-Yong Ok; syok@hknu.ac.kr
}

Received 23 May 2018; Accepted 28 August 2018; Published 1 October 2018

Academic Editor: Emanuele Reccia

Copyright (c) 2018 Seung-Yong Ok et al. This is an open access article distributed under the Creative Commons Attribution License, which permits unrestricted use, distribution, and reproduction in any medium, provided the original work is properly cited.

\begin{abstract}
One of the important goals of structural health monitoring is to identify structural damage using measured responses. However, such damage identification is sensitive to noises in the response measurements. Even a small change in the measurement may result in a significantly biased damage assessment. The goal of this paper is to expand the multiobjective optimization approach developed for robust damage identification in order to facilitate its applications to more realistic bridge damage identification problems. Specifically, a benchmark problem on highway bridges, developed under the auspices of International Association for Bridge Maintenance and Safety (IABMAS), is investigated. Various issues regarding sensor noises, multiple measurements, and loading scenarios are addressed to improve the robustness of bridge damage identification. A major finding from this study is that the stochastic process of Pareto optimal solutions obtained in a single run not only captures the actual damage locations successfully but also provides useful information such as damage-detected ratio on the potential candidates for damage to be inspected on site. Moreover, it is shown through the success, failure, and partial detection rates that the robustness of the proposed approach can be improved by using appropriate excitation scenarios and multiple sets of measurement data.
\end{abstract}

\section{Introduction}

Structural health monitoring involves identification of damage in the target structure using measured responses. One of the challenges in such damage identification is to deal with uncertainties caused by noises in the sensor or temporal variability of the measurement. The first step to overcome this challenge is to improve the sensing aspect. Obviously, the most suitable sensors should be selected for the type of damage to detect. The locations of sensors need to be optimized so as to maximize information gain [1]. Sometimes a network of sensors is deployed with fault-tolerance [2], such that meaningful measurements are obtained even with noises or failures of a few sensors.

By improving the sensing aspect, uncertainties in the measurement can be minimized but generally cannot be eliminated. Since damage identification problems are inverse problems, they are highly sensitive against the uncertainties in the measurement. That is, for given output responses (=sensor measurements) under specified loads, changes in structural properties (=damage state) are inversely identified. Such an inverse problem is inherently prone to errors in the response measurements because even a small change in the response may cause the inverse analysis to provide significantly different damage assessment results.

Various approaches have been proposed to deal with the uncertainty issue, such as the Bayesian probabilistic approach [3], regularization [4], fuzzy sets [5], substructuring [6], reliability index [7], and multiobjective optimization [8]. Most of the damage identification problems are formulated as the single-objective optimization problem, whereby the goal of the optimization is to identify the damage state that 
best describes the observed sensor measurement. Some researchers employed the multiobjective optimization approach in order to improve the damage identification performance [8-12]. In particular, the multiobjective approach is shown to improve the robustness of damage identification when noise in the measurement is relatively high, or when available measurement is insufficient [8]. To achieve robustness of damage detection, the method should not miss the existence of damage even with the uncertainties in the measurement. In addition, preferably, the method should not show falsenegative detection. To achieve these, the multiobjective approach first obtains Pareto optimal solutions and then applies stochastic processes to the solutions.

The premise of the multiobjective approach is that optimization using multiple sets of measurements will improve the accuracy of the damage detection, provided that the noise or error in the measurement does not have intrinsic biases. Compared to the $N$ repetitions of single-objective optimization, the multiobjective optimization with $N$ Pareto solutions showed significantly better performances because the Pareto solutions, based on the nondomination framework, help prevent convergence to an incorrect solution [8]. On the other hand, repetitions of single-objective optimization tend to all converge to incorrect solutions, when there are high levels of noise or error in the measurements. Therefore, processing $N$ solutions from single-objective optimization is not as accurate as $N$ Pareto optimal solutions.

The goal of this paper is to expand the multiobjective approach by the authors [8] by applying the method to more realistic bridge damage identification problems, for which $N$ Pareto solutions are processed statistically to investigate the possibility of damage at each location, and success/failure rate of detection or partial detection rate. Previously, the multiobjective approach was shown to improve the robustness of damage detection of a simply-supported truss structure [8]. This paper applies the method to a realistic problem in terms of both the target structure and the damage scenarios. The damage detection benchmark example investigated in this paper was developed by Caicedo et al. [13] under the auspices of International Association for Bridge Maintenance and Safety (IABMAS). Details of the example structure will be first discussed in the next section.

\section{Benchmark Problem}

2.1. Benchmark Bridge Model. The benchmark problem represents behavioral characteristics of a generic deck-onbeam-type bridge. This type of bridge constitutes a significant portion of the U.S. bridge inventory. Details of the benchmark model as well as the finite element model can be found at the benchmark bridge website [14]. Figure 1 shows the finite element model of the benchmark bridge, which has two spans. The longitudinal length of the model is $5.5 \mathrm{~m}$, with transverse beams spaced at $0.9 \mathrm{~m}$. The overall dimension of the model is $5.5 \mathrm{~m} \times 1.8 \mathrm{~m} \times 1.1 \mathrm{~m}$. The crosssection was chosen as $\mathrm{S} 3 \times 5.7$ so that the model structure can represent typical short-to-medium span highway bridges in terms of their modal frequencies, deflections, rotations, stresses, and strains [13].
This study uses the numerical model provided in the benchmark bridge website [14]. The numerical model was used in order to study the effect of the sensor noise systematically. The model simulates randomly generated noise in the sensor measurements. Although the default sensor noise in the benchmark problem is a uniformly distributed random noise with $0.1 \%$ of peak response, we vary the noise level up to $10 \%$ to test and demonstrate the robustness of our method. In order to simulate the uncertainties in the loads, a normally distributed random noise with zero mean and standard deviation as large as $0.2 \%$ of the loading magnitude is added to each force value.

The numerical phase allows researchers to simulate static or dynamic tests, along with several different types of sensors. During the static test, the sensors measure displacement and strain, whereas acceleration and strain are measured during the dynamic test measures. The proposed multiobjective optimization approach is applicable to both static and dynamic tests, but the illustrative example of this paper is based on the static tests.

2.2. Test Setup and Damage Scenarios. Figure 2 illustrates the installation layout of the exciters and sensors. For measurement purpose, a total of 14 displacement transducers are used to measure the deflection of the bridge girder at each connection node. For excitation purpose, we consider a total of 4 excitation points which correspond to the center points of the two longitudinal beams at each side of the bridge. We consider the two sets of loading scenarios as listed in Table 1, which will be used for two objective functions to be minimized in the multiobjective optimization approach.

The first loading scenario (LS1) has two sets of loading conditions. The first load set (Lset1) is to apply the pair of loads with 2,500 $\mathrm{N}$ on nodes 69 and 132, and the second load set (Lset2) is to apply the pair of loads with 2,500 $\mathrm{N}$ on nodes 90 and 111. For the applied loads, vertical displacements are recorded at 14 locations shown in Figure 2. The measurement data collected from each load set are used to construct the corresponding objective function. Here, random noises are incorporated into the load and measurement data in order to simulate the actual experimental environment. Therefore, if the tests are repeated several times, the load and displacement data obtained for each test will vary randomly each time depending on generated samples representing the noises in load and displacement.

The second loading scenario (LS2) also has two sets of loading conditions where the magnitudes of the loads $(2,500 \mathrm{~N})$ are the same as LS1, but the loading combinations are different. The first load set (Lset1), i.e., the pair of loads on nodes 69 and 111, is used to construct one objective function while the second objective function is determined by the two loads on nodes 90 and 132 in the second load set (Lset2). The noises in the load and sensor data follow the same distribution as LS1.

Among the damage scenarios provided in the benchmark, we consider the damage scenarios presented in Table 2 . The description of damage scenarios in the help file accompanying the numerical model [14] is adapted in the context of this study. Each scenario has multiple levels of damage. 


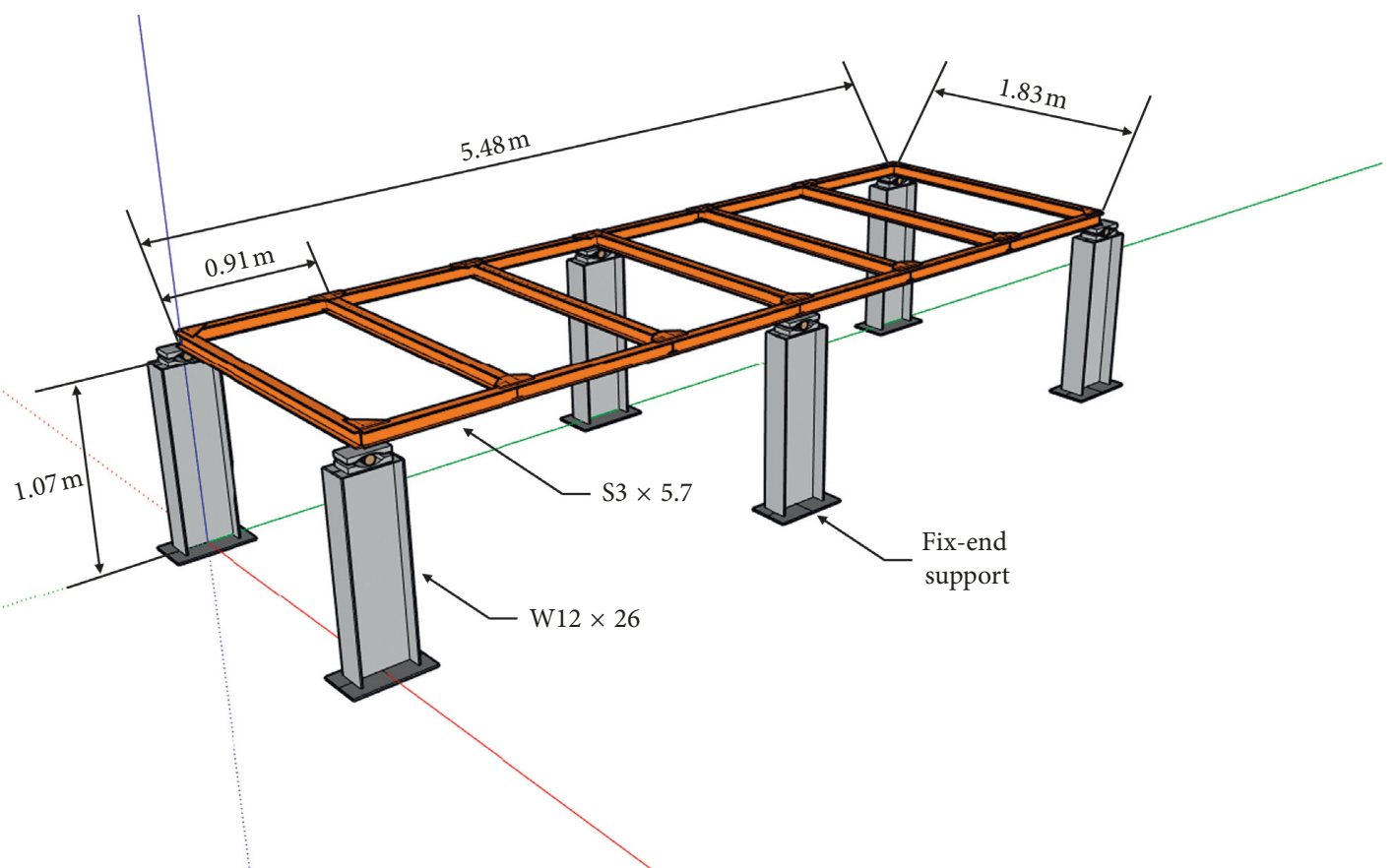

FIgURE 1: Two-span continuous bridge based on benchmark problem.

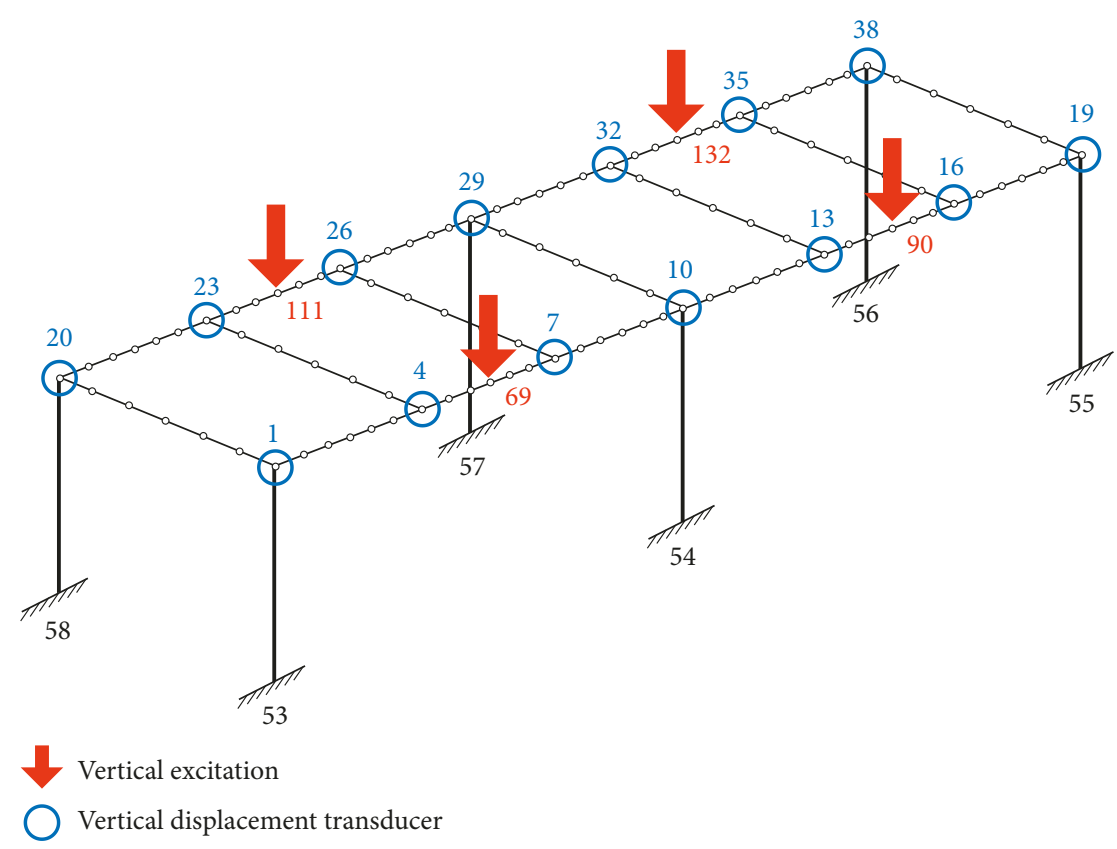

FIgURE 2: Installation layout of exciters and sensors.

TABLE 1: Loading scenarios.

\begin{tabular}{lccc}
\hline Case & Load set & Nodes & Objective functions \\
\hline \multirow{2}{*}{ LS1 } & Lset1 & 69,132 & To construct objective 1 \\
& Lset2 & 90,111 & To construct objective 2 \\
\hline \multirow{2}{*}{ LS2 } & Lset1 & 69,111 & To construct objective 1 \\
& Lset2 & 90,132 & To construct objective 2 \\
\hline
\end{tabular}

In order to examine the robustness of the proposed approach, the level of the sensor noise is varied over a wide range: $0.1,0.5,1.0,2.0,3.0,5.0$, and $10.0 \%$ of the peak displacement. One, five, and ten sets of measurements are separately tried for both load cases to examine the effects of loading scenarios, sensor noises, and repeated measurements.

\section{Multiobjective-Optimization- (MOO-) Based Damage Identification}

3.1. MOO-Based Formulation for Damage Identification Problems. The goal of structural damage identification is to detect the damage state given the change observed in the measurement. For this purpose, the problem is often 
TABle 2: Damage scenarios.

\begin{tabular}{lccc}
\hline Scenario & Case & Locations & Damage scenarios \\
\hline DS1 & Single & Node 7 & Nodes 7,26 \\
DS2 & Single & Node 19 & $\begin{array}{c}\text { Damage in connection between longitudinal and } \\
\text { transverse beams by removing connection plates and } \\
\text { bolts at joint, in order to simulate the local loss of } \\
\text { stiffness at the connection }\end{array}$ \\
\hline
\end{tabular}

formulated in the context of single-objective optimization where the objective function is defined as the weighted sum of the differences between measured responses and modelestimated responses, which are observed in multiple sets of measurements. As pointed out in the previous study [8], however, the accuracy and robustness of the solution of the single-objective optimization problem are inevitably influenced and significantly fluctuated by the selection of the weighting factors and uncertain noises in measurements. Therefore, it is not straightforward to construct a singleobjective function which successfully works for multiple sets of measurements. In order to overcome this difficulty, we adopt a weight-free bi-objective optimization approach that employs the following vector form of two objective functions:

$$
\min \mathbf{F}(\mathbf{x})=\left\{\begin{array}{l}
\sum_{i=1}^{N_{\mathrm{m}}}\left\|\mathbf{u}_{i}^{\mathrm{p}}(\mathbf{x})-\mathbf{u}_{i}^{\mathrm{m}}\right\|_{\text {Lset1 }}^{2} \\
\sum_{i=1}^{N_{\mathrm{m}}}\left\|\mathbf{u}_{i}^{\mathrm{p}}(\mathbf{x})-\mathbf{u}_{i}^{\mathrm{m}}\right\|_{\text {Lset2 }}^{2}
\end{array}\right\}
$$

where $\mathbf{u}$ is a $14 \times 1$ vector of vertical displacement of the bridge; the superscripts $\mathrm{p}$ and $\mathrm{m}$ represent prediction and measurement, respectively; the subscript $i$ denotes the index of the repeated measurements; $N_{\mathrm{m}}$ is the number of the repeated measurements; and the subscript Lset1 and Lset2 represent the two sets of loadings of a specific loading scenario given in Table 1 . The measured response $\mathbf{u}^{\mathrm{m}}$ is the a priori given information obtained from the simulation of the benchmark bridge model under Lset1 and Lset2, and the predicted response $\mathbf{u}^{\mathrm{p}}$ is computed from the numerical model, as follows:

$$
\mathbf{u}^{\mathrm{p}}(\mathbf{x})=\mathbf{K}(\mathbf{x})^{-1} \mathbf{F},
$$

where $\mathbf{K}(\mathbf{x})$ is the global stiffness matrix of the structure and $\mathbf{F}$ is the global force vector. It should be noted that the stiffness matrix is a function of the design variable vector $\mathbf{x}$. The damage in the structure can be described by changes in the design variables. The procedure of minimizing the biobjective function is then to explore the damage in the structure, which is represented by the global stiffness matrix that makes the displacement predictions of the numerical model as close as possible to the response measurements.

As explained in [8], a solution to the conventional single objective optimization approach can easily converge to an incorrect solution due to the bias caused by the sensor noise. On the other hand, the multiobjective optimization approach can improve its accuracy because the conflicting feature in multiple objectives helps to prevent convergence to an incorrect solution. Also, it is demonstrated in [8] that the nondomination scheme in the multiobjective optimization effectively discourages convergence to an incorrect solution and enables the low level of false-negative solutions for robust damage detection.

3.2. GA-Based Optimization Scheme. The presence of multiple objectives in a problem, in principle, produces a set of optimal solutions, referred to as Pareto optimal solutions, rather than a single optimal solution. This is due to the nondomination concept in minimizing the two objective functions simultaneously. For example, let us consider $\min \mathbf{F}=\left[f_{1}(\mathbf{x})\right.$ and $\left.f_{2}(\mathbf{x})\right]$. Since a global optimal solution minimizing both functions does not exist in general engineering problems, it is inevitable to have two solutions $\mathbf{x}_{1}$ and $\mathbf{x}_{2}$ satisfying the following relations:

$$
\begin{aligned}
& f_{1}\left(\mathbf{x}_{1}\right)<f_{1}\left(\mathbf{x}_{2}\right), \\
& f_{2}\left(\mathbf{x}_{1}\right)>f_{2}\left(\mathbf{x}_{2}\right) .
\end{aligned}
$$

This relation itself never guarantees that $\mathbf{x}_{1}$ is better than $\mathbf{x}_{2}$ or vice versa. These two solutions are nondominated to each other. Therefore, a simultaneous minimization of multiple objective functions essentially leads to finding many nondominated optimal solutions. Searching for these mutually conflicting optimal solutions requires a multipoint parallel comparison which is well suited for genetic algorithm (GA). Accordingly, there have been various studies on the application of genetic algorithm to the multiobjective optimization problem in the field of structural control and health monitoring [15-17]. In this study, we have incorporated NSGA-II [18], one of the most widely used multiobjective optimization techniques into the structural damage detection problem.

For multipoint parallel searching, the NSGA-II algorithm first constructs a population of multiple encoded individuals, i.e., design variables with damage information, through the random generation. Each individual is evaluated in terms of objective functions in Equation (1). Then, based on the function values, the algorithm identifies the first level of the nondominated solutions which correspond to the 
individuals that are not dominated by any other individuals in the population. The first rank is identically assigned to the first level of the nondominated individuals. Except for the first-level individuals, the nondomination comparison is repeated to assign the rank values to all remaining individuals. The individuals with the same rank are additionally evaluated by using crowding distance which is the density measure of a particular individual, i.e., how many other individuals with the same nondomination level exist closely around the particular individual. With the rank value and the crowding distance, NSGA-II selects the parent individuals to construct the population of the next generation. The solutions with lower rank are most preferably chosen. Thus, the first-level solutions are selected first, and if the number of the solutions is insufficient to construct the population of one generation, then the second- and third-level solutions are included into the parents. However, if the first-level solutions exceed the population of a generation, the solutions with larger crowding distance are preferred and those with lower crowding distance are excluded. This selection process guides the search towards the better diversity of Pareto optimal solutions and prevents the solutions from converging to incorrect solutions. Then, NSGA-II performs the typical GA operations such as crossover and mutation in order to generate the offspring individuals for the next generation of population. Then, the evaluation of rank and crowding distance is repeated for the offsprings, and so is the GA operation. Through these iterations up to a specified maximum number of generations, the population evolves towards the global Pareto optimal solutions as much as possible.

\section{Illustrative Example}

4.1. Problem Setup. For the proposed GA-based optimization approach, the design variables correspond to the locations of the damage, which reflects the local loss of stiffness at the connection and the support locking at the top of the bridge pier, finally converging to the measured responses through the change in the global stiffness matrix. For the two previously- described damage scenarios, there are a total of 14 damage locations for the connection damage DS1 (nodes 1, 4, $7,10,13,16,19,20,23,26,29,32,35$, and 38) and a total of 6 damage locations for the boundary damage DS2 (nodes 1, 10, 19, 20, 29, and 38). Accordingly, the design variables are encoded as chromosomes in GA code to represent 14 damage locations for the connection damages and 6 damage locations for the boundary damages, including no damage case. Since we will not know how many damage locations and what kind of structural damage occur, it is assumed that the damage can occur at up to 3 locations, regardless of the type of the damage. If the damage locations are assigned to 1 or 2 design variables, the remaining design variables will be defined to include no damage case $(=0)$. In this way, the 3 damaged and undamaged locations are assigned to each chromosome, and they are searched to best fit the measured displacements. The population of one generation was set to 50 , and the multiobjective optimization process was performed till the maximum number of the iteration for the entire generation reaches 100 .

4.2. Effects of Loading Scenarios. In order to investigate the effects of loading scenarios on the damage detection performance, the proposed approach has been applied to the damage scenarios in Table 2. The loading conditions were LS1 and LS2 shown in Table 1, and the excitation was applied only once. Therefore, only one set of 14 displacement data was used for damage detection. As a result of applying the proposed approach, a total of 50 Pareto optimal solutions were obtained for each case. Since each design variable contains 3 damage locations including no-damage case, the Pareto solutions consist of 150 "damage" or "no-damage" information in total. Based on this information, the possibility of damage at each location is illustrated in Figures 3-6. Each figure shows the comparison of the optimization results obtained under the LS1 and LS2 excitation conditions. The horizontal axis corresponds to the location of the detected damages, where the connection damages are referred to as $\mathrm{C} 01, \mathrm{C} 04, \ldots, \mathrm{C} 38$, and the boundary damages are denoted as B01, B10, .., B38. The vertical axis represents the ratio of the number of the detected damages per location to the total number of the damage cases detected by the proposed approach. Therefore, the vertical value indicates the possibility of damage at each location.

Figure 3 shows the case of single damage in DS1. Since the connection damage occurs at node 7 in DS1, the true solution is C07. It is shown that the true solution C07 was captured successfully by both LS1 and LS2. However, the use of LS1 and LS2 shows a slight difference in predicting the possibility of the location-specific damage. LS1 provides $\mathrm{C} 07$ as the most probable solution with the highest ratio, while it also offers a variety of potentially feasible solutions with the lower ratio than $\mathrm{C} 07$. For example, $\mathrm{C} 19$ and $\mathrm{C} 20$ have the second highest ratio; $\mathrm{C} 10, \mathrm{C} 16$, and $\mathrm{C} 38$ have the third highest ratio; and so on. These solutions except for $\mathrm{C} 07$ correspond to false-positive solutions. In contrast, LS2 provides $\mathrm{C} 20$ as the most likely solution and $\mathrm{C} 07$ as the second-highest solution, and two more solutions are detected additionally with fairly low ratio values. When considering the results of both methods simultaneously, $\mathrm{C} 07$ and C20 can be determined as the potential candidates for damage to be inspected on site.

Figure 4 displays the case of multiple damages in DS1 where the true solutions correspond to $\mathrm{C} 07$ and C26. In this case, both LS1 and LS2 successfully detect the two damage cases at $\mathrm{C} 07$ and $\mathrm{C} 26$ with the equally highest ratio. On the other hand, LS1 additionally captures the damage at C38 with the same highest ratio, whereas LS2 detects C19 and C20 with lower ratio.

Similar results are observed for the DS2 cases as shown in Figures 5 and 6 . Figure 5 corresponds to the case of single damage in DS2 where the true solution is B19. Both LS1 and LS2 show successful detection of the true solution. Similar to the previous results, additional false-positive solutions are also being detected. As shown in Figure 6, both LS1 and LS2 have successfully detected true solutions for multiple damage 


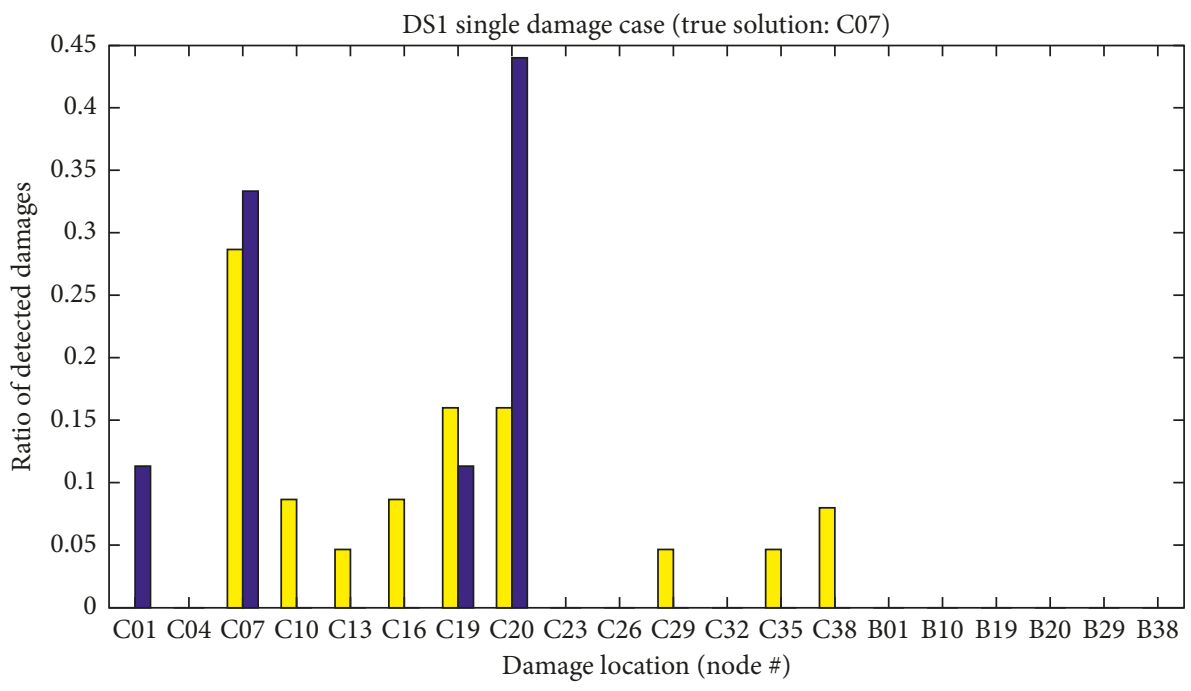

Loading scenario 1

Loading scenario 2

FIGURE 3: Damage identification results for DS1 single damage scenario.

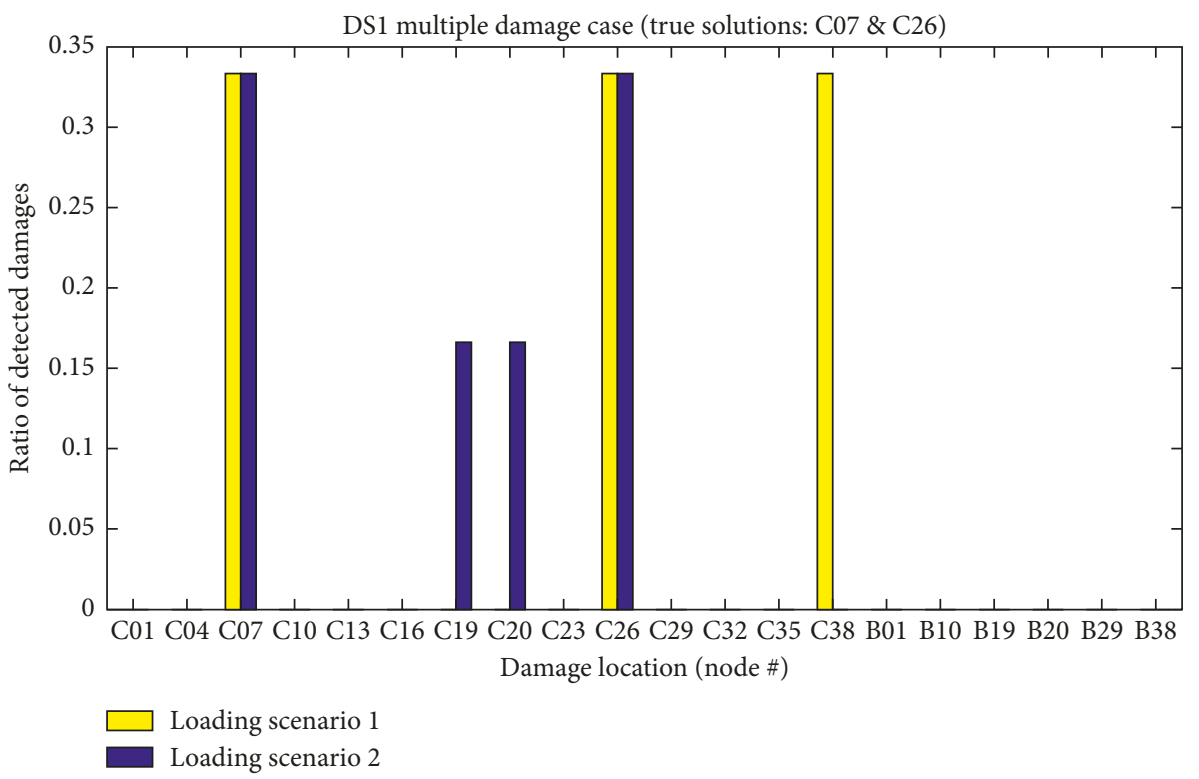

FIGURE 4: Damage identification results for DS1 multiple damage scenario.

scenarios in DS2 as well, and, similarly, additional damage locations are predicted as C16 by LS1 and C32 by LS2.

These results demonstrate that when statistically processing all 50 Pareto optimal solutions obtained by the proposed approach, the results can successfully include the locations where the actual damage occurred. More specifically, the ratio of the number of the detected damage cases per location to those detected by the proposed approach can provide us with the information on potential candidates for damage to be inspected on site, including the actual damage and false-positive damage, although there exist some differences in performance depending on the loading conditions and the scale of the damage. Next, let us investigate the effect of the sensor noise on the detection performance of the proposed approach.
4.3. Effects of Sensor Noises. The performance of the damage identification technique is often evaluated by the success rate of detecting the true damage cases. That is, the performance of the detection approach can be evaluated as an index indicating how many times the actual damage cases are detected among the total execution numbers of the approach. In this study, unlike the existing methods, we obtained 50 Pareto optimal solutions simultaneously in a single run. Therefore, we newly define the success rate index of the true damage detection as the ratio of the number of Pareto optimal solutions that correctly detected actual damage, among all Pareto optimal solutions.

Here, we will investigate the effects of sensor noises on the damage detection performance of the proposed approach. For this purpose, the proposed approach has been 


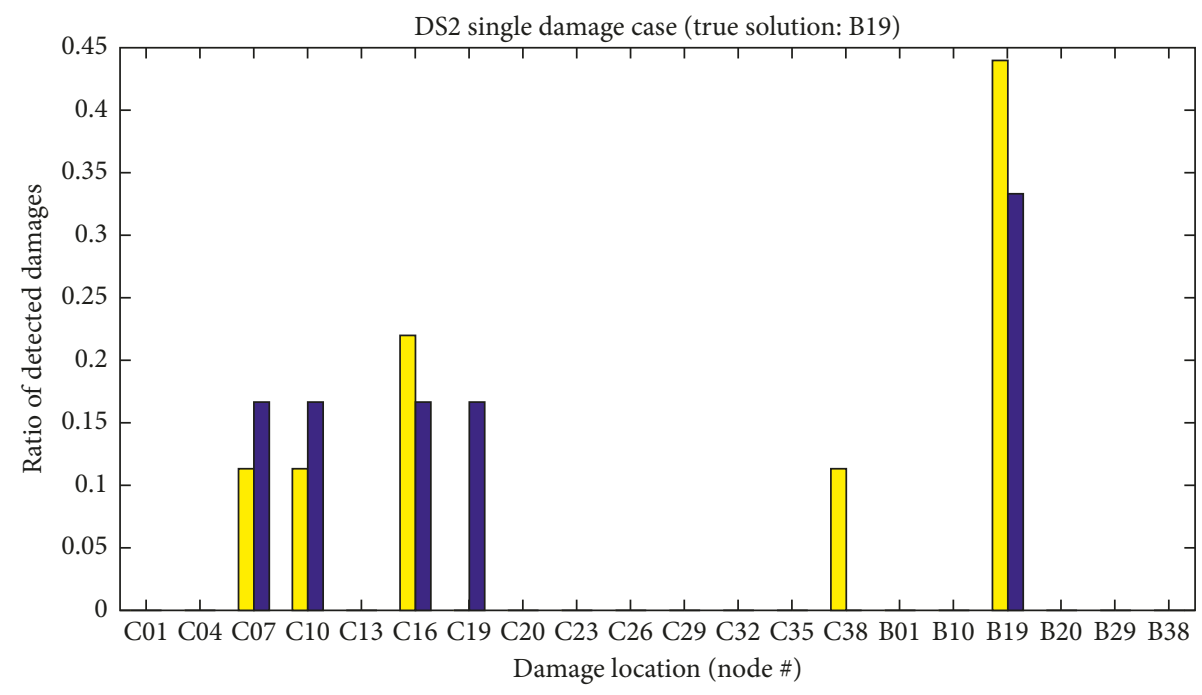

Loading scenario 1

Loading scenario 2

FIGURE 5: Damage identification results for DS2 single damage scenario.

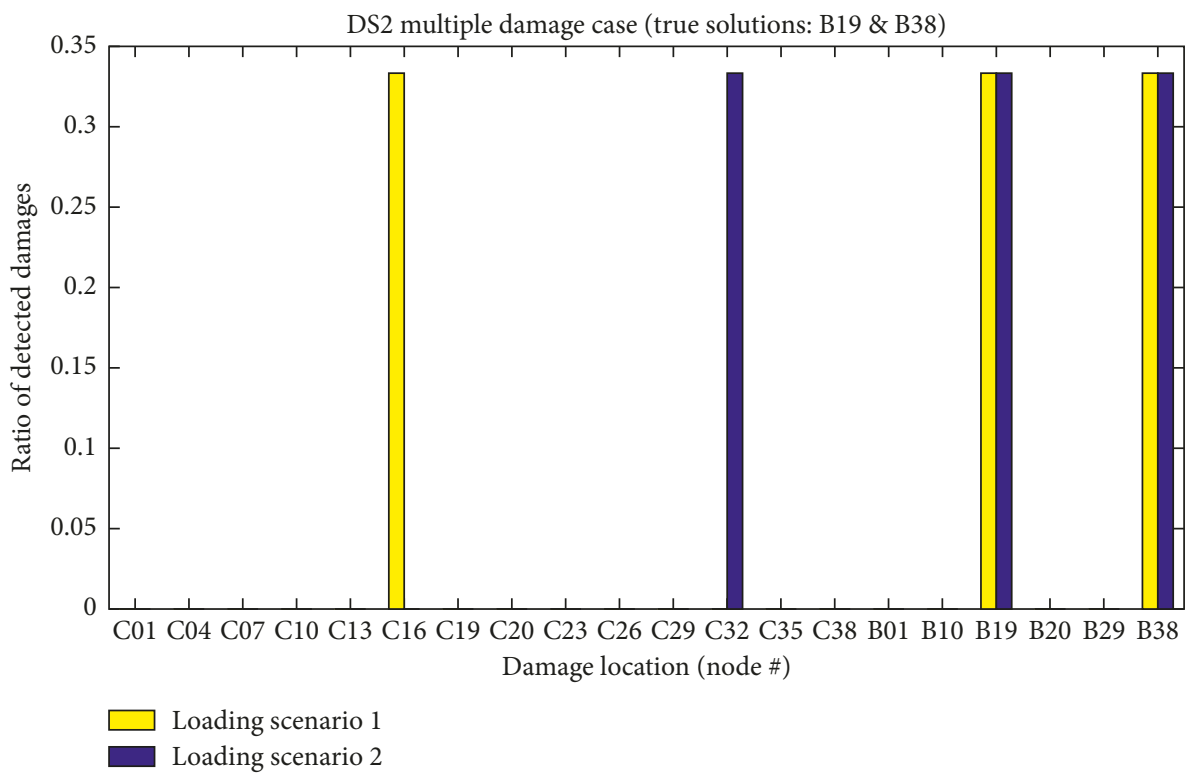

FIgURE 6: Damage identification results for DS2 multiple damage scenario.

applied repeatedly to the same damage scenarios by varying the level of sensor noise from $0.1 \%$ to $10 \%$. The results are illustrated in Figures 7 and 8. Figures 7 and 8 show the comparative results between the 4 damage scenarios under the LS1 and LS2 excitations, respectively. The horizontal axis corresponds to the noise level in the sensor measurements, and the vertical axis represents the success rate of the damage detection among the 50 Pareto optimal solutions.

As can be shown in Figure 7, the boundary damages consisting of the single and multiple damage cases in DS2 are all successfully identified with $100 \%$ certainty regardless of the level of the sensor noises. However, for DS1, there are cases where the success rate does not reach $100 \%$ when the sensor noises are present, e.g., $0.1 \%, 1.0 \%$, and $10 \%$ for single damage, and $1.0 \%, 5.0 \%$, and $10.0 \%$ for multiple damage locations. In particular, in case of multiple damage locations of DS1, the success rate was $0 \%$, for which further investigations will be provided later (Figure 9). This implies that all Pareto optimal solutions fail to detect the two damage locations at nodes C07 and C26. Overall, the performance on the multiple damage detection is further degraded, compared to the case of the single damage detection. This tendency is similar for the case of LS2 excitation conditions, as shown in Figure 8 . In the case of multiple damage locations in DS1, the success rate tends to decrease with increasing sensor noises while showing some fluctuations. On the other hand, Figure 8 shows that the single damage is not missed even if sensor noises increase up to 


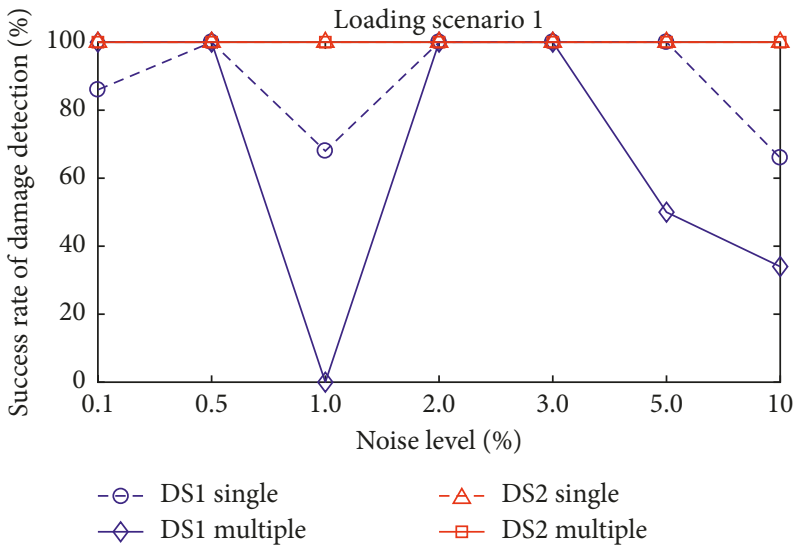

FIGURE 7: Variations in success rate of damage detection under LS1 with respect to sensor noise levels.

3.0\%. Unlike LS1, the success rate of DS2 multiple damages does not show $100 \%$. Nonetheless, when comparing Figures 7 and 8, the success rate of damage detection according to sensor noise level is more stable under LS2 excitation condition than LS1. This is due to the fact that a load set 1 of LS2 is acting at nodes 69 and 111, which are close to nodes 7 and 26 of the two damage locations. By doing this, the excited responses show a clear difference from the response of the bridge with no damage, which leads to more stable success rate of damage detection.

In order to examine the results of Figures 7 and 8 in which the success rate does not reach $100 \%$, we define two additional indices, i.e., failure rate and partial detection rate. The failure rate index is defined as the percentage of the Pareto optimal solutions which fail to detect the true solution. This failure rate index indicates that the proposed approach completely fails to detect the actual damage. When the damages occur at multiple locations, it means that no single damage is detected. On the other hand, in case of multiple damage case, there also exists a possibility that the method only finds partial damage among the multiple damage cases. Accordingly, the partial detection rate is additionally defined as the percentage of the Pareto solutions which detect only one damage among the two damage cases. As already observed in Figures 7 and 8, the success rates of the DS2 single damage case have all reached 100\% under both LS1 and LS2 for all sensor noise levels. Therefore, except for the DS2 single damage case, Figures 9-11 represent the contributions of success, failure, and partial detection rate indices for the cases of DS1 single damage, DS1 multiple damage, and DS2 multiple damage, respectively.

In the single damage case of Figure 10, the partial detection rate cannot appear. Under the LS2 condition, the failure rate only appears with the sensor noises of $5.0 \%$ and $10.0 \%$, and the success rate remains stable with the sensor noises of $0.1 \% \sim 3.0 \%$. On the contrary, under the LS1 condition, the failure rate appears irregularly with the sensor noises of $0.1 \%, 1.0 \%$, and $10.0 \%$.

As shown in Figures 9 and 11, in the case of multiple damage cases in DS1 and DS2, the failure rate actually does not appear at all. Instead, if the $100 \%$ success rate is not achieved, the partial detection rate is filling the remaining percentage.

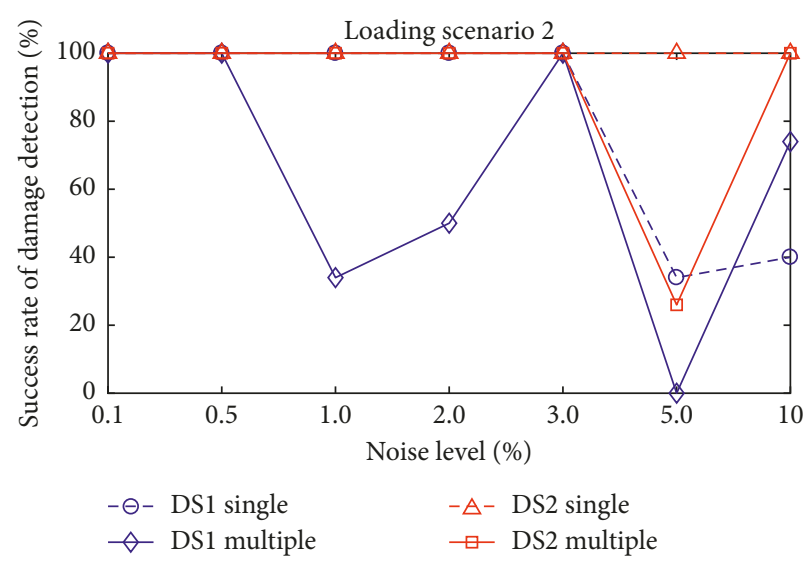

FIGURE 8: Variations in success rate of damage detection under LS2 with respect to sensor noise levels.

Further, Figures 7-11 do not show a clear tendency to converge along with the increase in sensor noise in terms of success, failure, and partial detection rate indices of damage detection. In other words, as the noise level increases, the indices do not tend to decrease steadily but fluctuates instead. This is because these results were obtained with only one measurement data. Therefore, it is inferred that irregularity or biasedness of noise generation applied to 14 measurement data has more significant influence than sensor noise level itself. So, we will investigate the effects of measurement data on the detection performance in the next section.

4.4. Effects of Measurement Data. Let us now consider the case of using two different sets of measurement data, i.e., 5 sets and 10 sets, as input information for the optimization process. When 5 sets of measurements are used, 14 displacements are repeatedly measured 5 times using the same loading sets. At each time, the noises of loads and sensors are randomly generated under the prescribed distributions. As a result, the variations of success rate of damage detection with respect to measurement data are comparatively illustrated in Figure 12 (DS1 single damage), Figure 13 (DS1 multiple damage), and Figure 14 (DS2 multiple damages). 


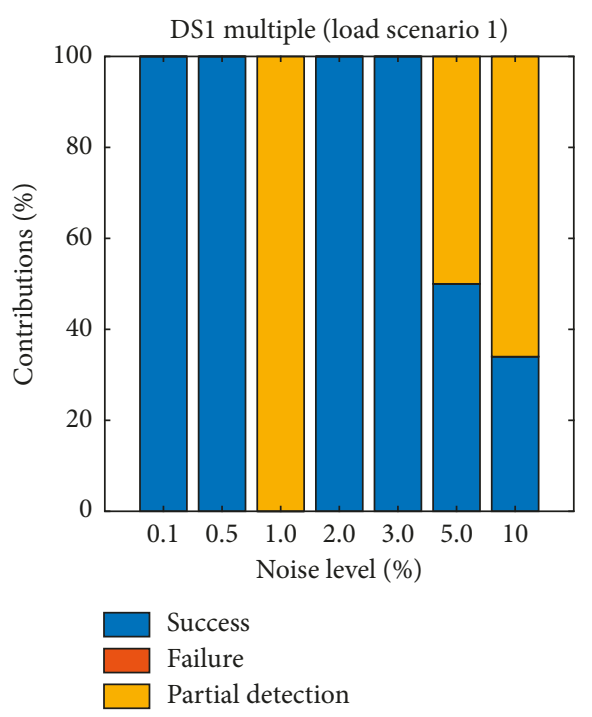

(a)

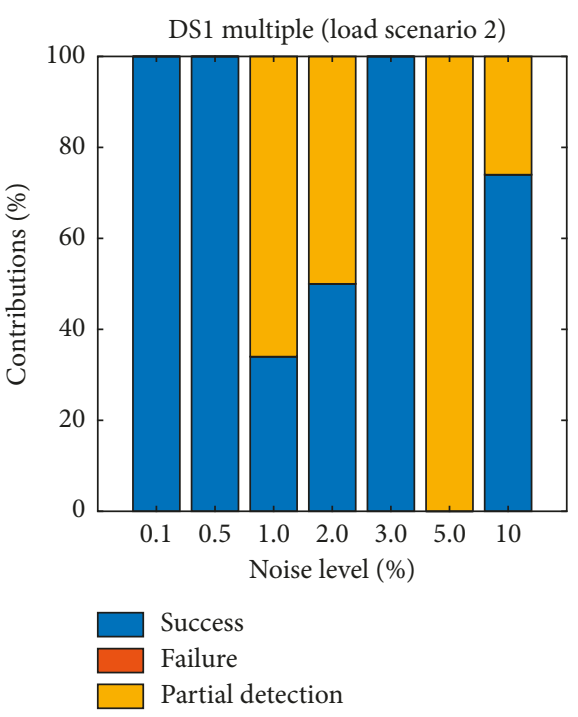

(b)

FIgURE 9: Contributions of success, failure, and partial detection rate indices for DS1 multiple damage case.

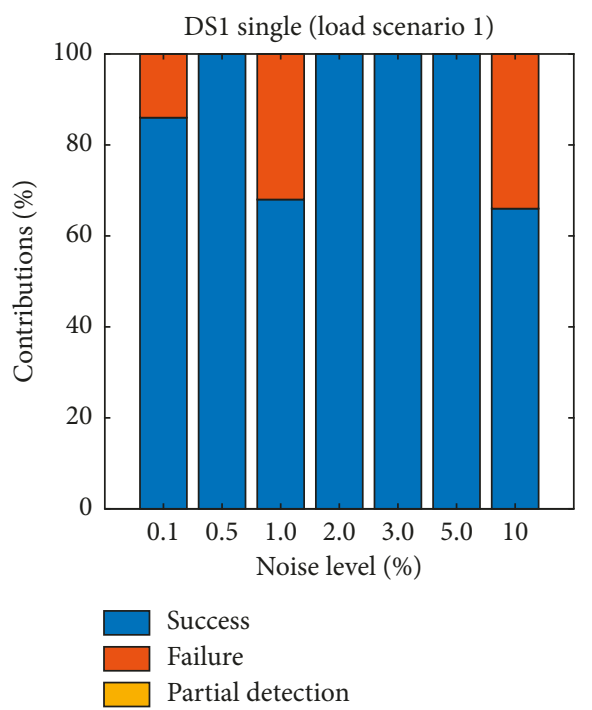

(a)

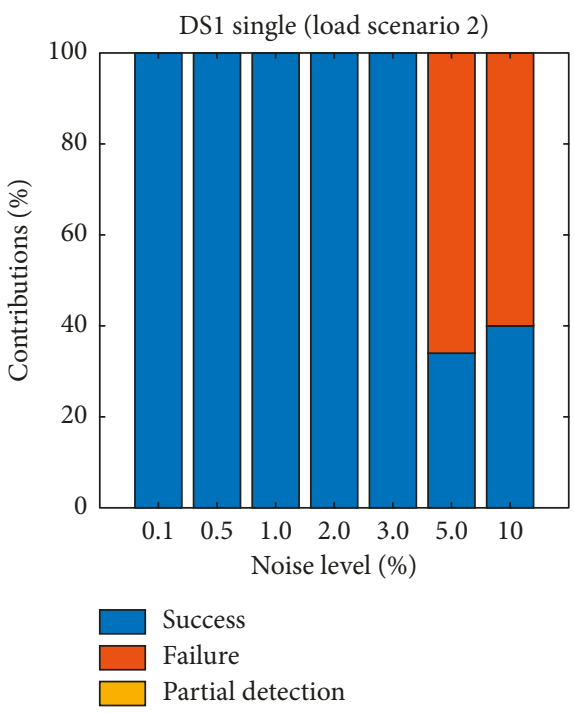

(b)

FIGURE 10: Contributions of success, failure, and partial detection rate indices for DS1 single damage case.

Although there are still some fluctuations depending on the sensor noise, it is confirmed that the success rates of damage detection are generally shifted to the right according to the sensor noise of the horizontal axis by repeatedly using the measurement data. This implies that the use of multiple measurement data helps improve the success rate of damage detection against the increase in sensor noise. In addition, in case of DS1 multiple damages, the success rate has been evaluated as $0 \%$ for the sensor noises of $0.5 \%$ and $10.0 \%$ under LS1 condition. As shown in Figure 13, even if using 5 sets of measurement data, the success rate is computed to be $0 \%$ for the sensor noises of $1.0 \%$ under LS1 and 5.0\% under LS2. Even if the success rate is $0 \%$, it does not necessarily mean that the failure rate is $100 \%$. Instead, it actually means that the partial detection rate of identifying only one of the two damage cases becomes $100 \%$. Anyway, the use of 10 measurement data significantly improves the success rate up to $100 \%$ under the sensor noises of both $0.5 \%$ and $1.0 \%$ under LS1. The improvement is also observed for the sensor noise of 5.0\% under LS2. The use of 10 measurement data shows a success rate of $0 \%$ only for $10 \%$ sensor noise under LS1 condition, and otherwise it shows the success rates above a certain level. In particular, as shown in Figure 14, the use of 10 measurement data guarantees the $100 \%$ detection success rate for all sensor noises for multiple damages in DS2 under LS2 excitation conditions. These results demonstrate 


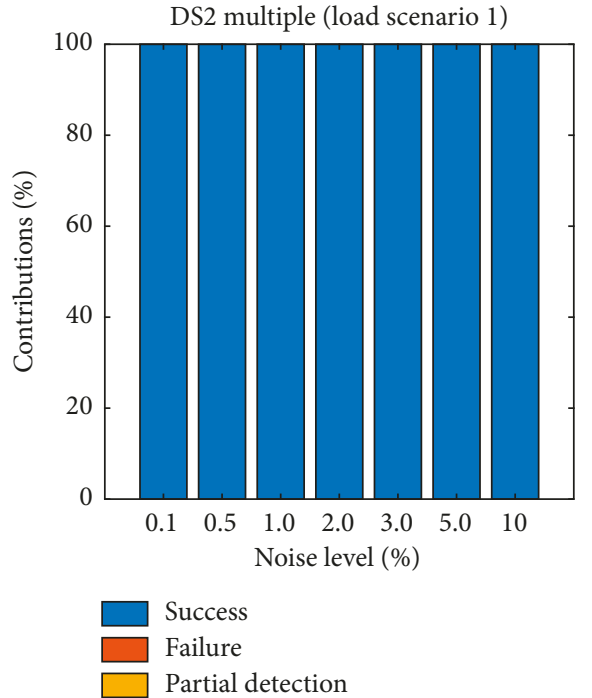

(a)

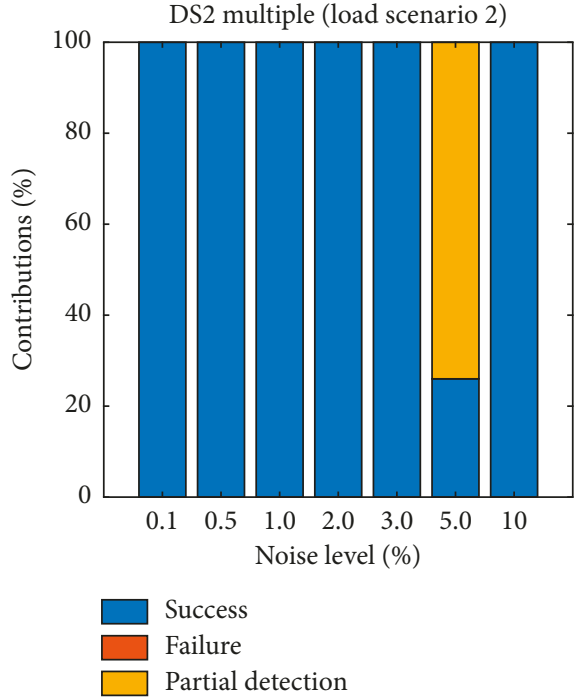

(b)

FIgUre 11: Contributions of success, failure, and partial detection rate indices for DS2 multiple damage case.

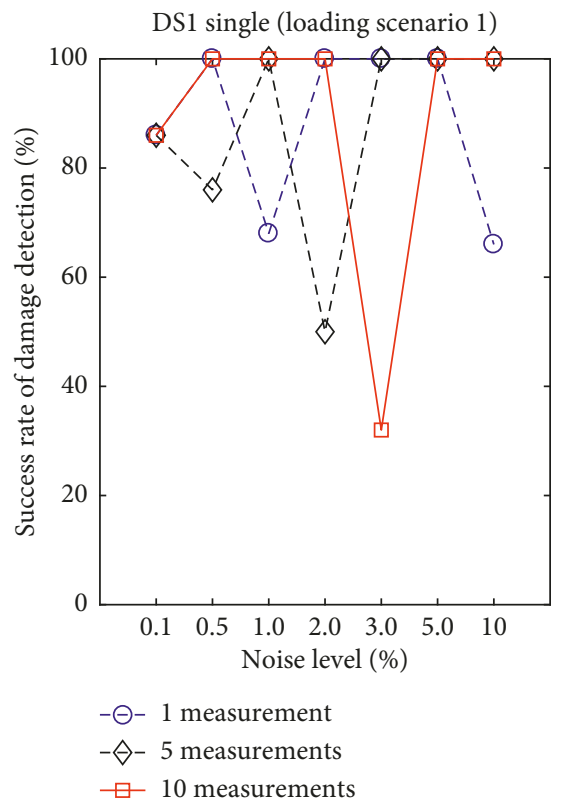

(a)

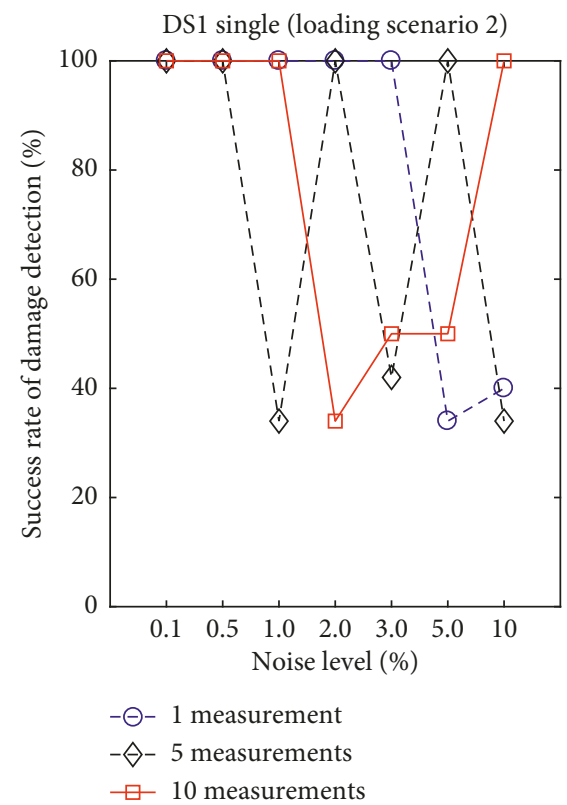

(b)

FIGURE 12: Variations in success rate for DS1 single damage with respect to measurement data. 


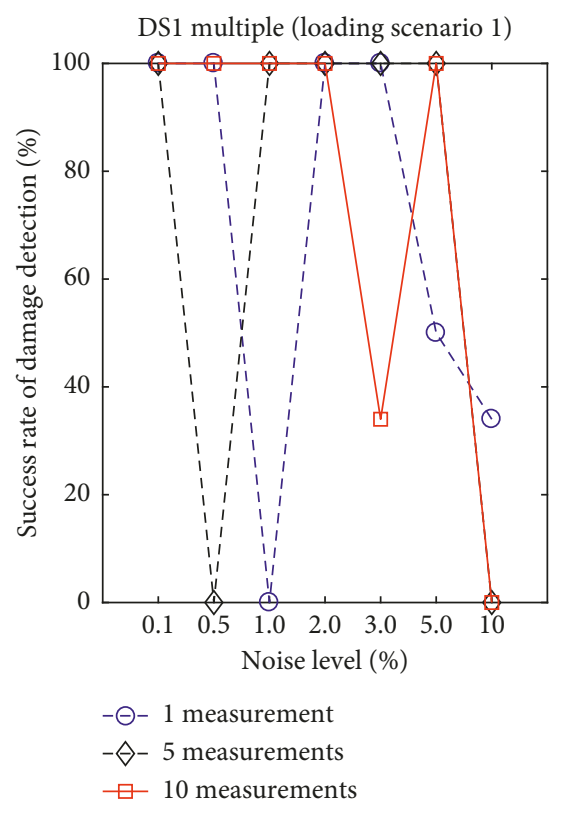

(a)

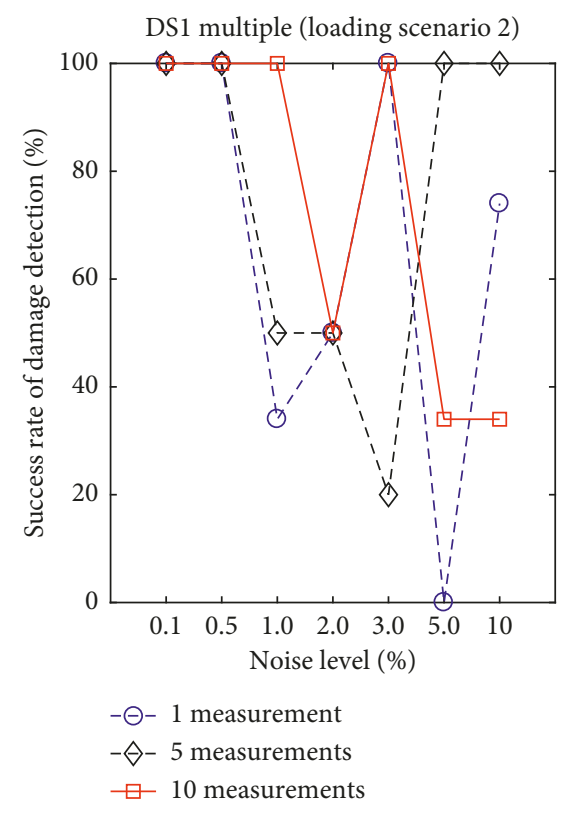

(b)

FIGURE 13: Variations in success rate for DS1 multiple damages with respect to measurement data.

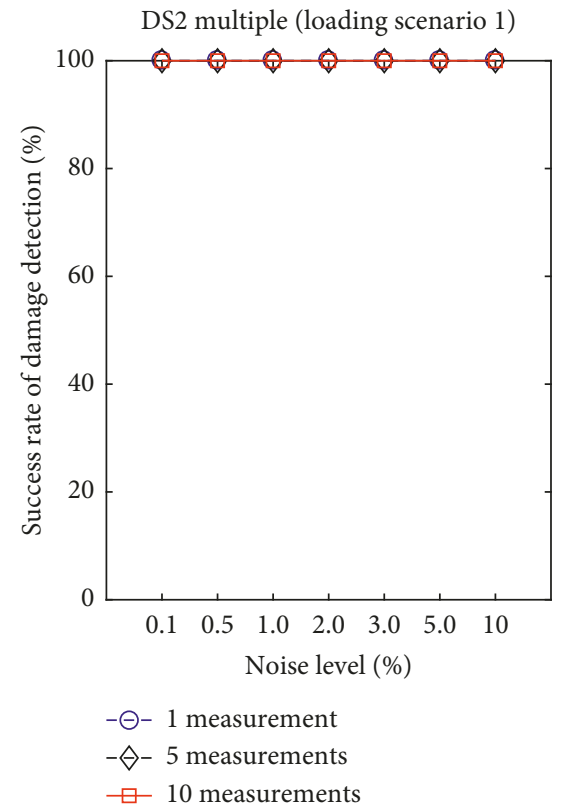

(a)

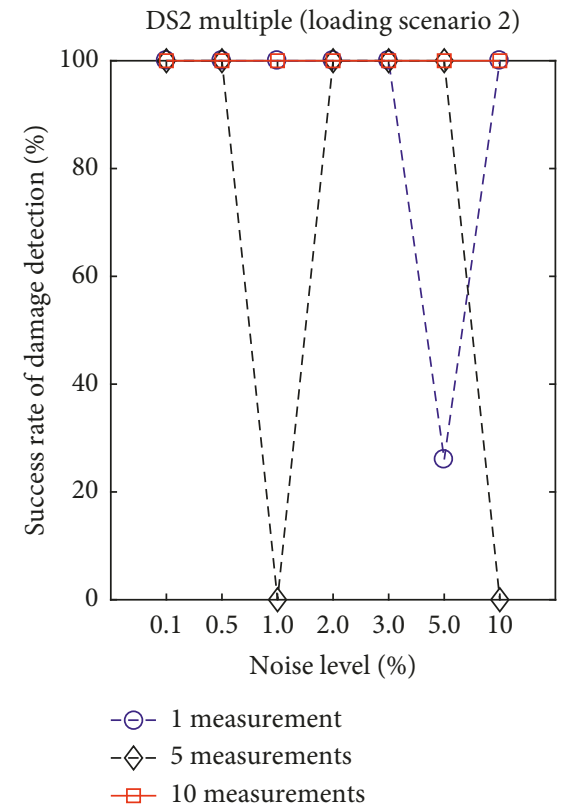

(b)

FIgURE 14: Variations in success rate for DS2 multiple damages with respect to measurement data.

that the more the measurement data is used, the more robust damage detection against the sensor noise is achieved.

\section{Conclusions}

This study expands a multiobjective damage identification approach through applications to a benchmark problem for highway bridges. The method utilizes multiple sets of measurements under different loading conditions in order to minimize adverse effects of uncertainty in the measurements on the results of damage identification. In order to demonstrate the robustness of the proposed approach, $N$ Pareto solutions obtained in a single run are processed statistically to show the possibility of damage per location and success, failure, and partial detection rates. The illustrative example demonstrates that the proposed approach is able to successfully 
capture not only the actual damage of the bridge but also the potential damage (false positive damage) that is inevitably observed in the inverse analysis of damage identification problem. This result provides us useful information such as damage-detected ratio on potential candidates for damage to be inspected on site. The success rate turned out to be improved possibly under the damage-specific loading scenario and further by using more measurement data. These results demonstrate that the robustness of the proposed approach against the sensor noise can be improved by increasing the measurement data under appropriate loading scenarios.

\section{Data Availability}

The data used to support the findings of this study are available from the corresponding author upon request.

\section{Conflicts of Interest}

The authors declare that they have no conflicts of interest.

\section{Acknowledgments}

This work was supported by Basic Science Research Program through the National Research Foundation of Korea (NRF) funded by the Ministry of Education (2017R1D1A1B04031418). The co-author Junho Song acknowledges the support from the Institute of Engineering Research at Seoul National University, South Korea.

\section{References}

[1] M. Meo and G. Zumpano, "On the optimal sensor placement techniques for a bridge structure," Engineering Structures, vol. 27, no. 10, pp. 1488-1497, 2005.

[2] M. Z. A. Bhuiyan, G. Wang, J. Cao, and J. Wu, "Deploying wireless sensor networks with fault-tolerance for structural health monitoring," IEEE Transactions on Computers, vol. 64, no. 2, pp. 382-395, 2015.

[3] M. W. Vanik, J. L. Beck, and S. Au, "Bayesian probabilistic approach to structural health monitoring," Journal of Engineering Mechanics, vol. 126, no. 7, pp. 738-745, 2000.

[4] H. W. Park, M. W. Park, B. K. Ahn, and H. S. Lee, "1-normbased regularization scheme for system identification of structures with discontinuous system parameters," International Journal for Numerical Methods in Engineering, vol. 69, no. 3, pp. 504-523, 2007.

[5] M. R. Taha and J. Lucero, "Damage identification for structural health monitoring using fuzzy pattern recognition," Engineering Structures, vol. 27, no. 12, pp. 1774-1783, 2005.

[6] K. V. Yuen and L. S. Katafygiotis, "Substructure identification and health monitoring using noisy response measurements only," Computer-Aided Civil and Infrastructure Engineering, vol. 21, no. 4, pp. 280-291, 2006.

[7] F. N. Catbas, M. Susoy, and D. M. Frangopol, "Structural health monitoring and reliability estimation: long span truss bridge application with environmental monitoring data," Engineering Structures, vol. 30, no. 9, pp. 2347-2359, 2008.

[8] S. Jung, S.-Y. Ok, and J. Song, "Robust structural damage identification based on multi-objective optimization," International Journal for Numerical Methods in Engineering, vol. 81, no. 6, pp. 786-804, 2009.
[9] Y. J. Cha and O. Buyukozturk, "Structural damage detection using modal strain energy and hybrid multiobjective optimization," Computer-Aided Civil and Infrastructure Engineering, vol. 30, no. 5, pp. 347-358, 2015.

[10] R. Perera, R. Marin, and A. Ruiz, "Static-dynamic multi-scale structural damage identification in a multi-objective framework," Journal of Sound and Vibration, vol. 332, no. 6, pp. 1484-1500, 2013.

[11] M. Wang and J. C. Brigham, "Assessment of multi-objective optimization for nondestructive evaluation of damage in structural components," Journal of Intelligent Material Systems and Structures, vol. 25, no. 9, pp. 1082-1096, 2014.

[12] E. Talic, A. Schirrer, M. Kozek, and S. Jakubek, "Multiobjective parameter identification of Euler-Bernoulli beams under axial load," Journal of Sound and Vibration, vol. 341, pp. 86-99, 2015.

[13] J. M. Caicedo, F. N. Catbas, M. Gul, and R. Zaurin, "Phase I of the benchmark problem for bridge health monitoring: numerical data," in Proceedings of the 18th Engineering $\mathrm{Me}$ chanics Division Conference of the ASCE, Virginia Tech, Blacksburg, VA, USA, June 2007.

[14] F. N. Catabas, J. M. Caicedo, and S. Dyke, "Benchmark problem on health monitoring of highway bridges," 2008, http://cee.ucf.edu/people/catbas/benchmark.htm.

[15] H. Liu, K. Xin, and Q. Qi, "Study of structural damage detection with multi-objective function genetic algorithms," Engineering Procedia, vol. 12, pp. 80-86, 2011.

[16] A. M. Raich and T. R. Liszkai, "Multi-objective optimization of sensor and excitation layouts for frequency response function-based structural damage identification," ComputerAided Civil and Infrastructure Engineering, vol. 27, no. 2, pp. 95-117, 2012.

[17] Y. J. Cha, A. Raich, L. Barroso, and A. Agrawal, "Optimal placement of active control devices and sensors in frame structures using multi-objective genetic algorithms," Structural Control and Health Monitoring, vol. 20, no. 1, pp. 16-44, 2013.

[18] K. Deb, A. Pratap, S. Agarwal, and T. Meyarivan, "A fast and elitist multi-objective genetic algorithm: NSGA-II," IEEE Transactions on Evolutionary Computation, vol. 6, no. 2, pp. 182-197, 2002. 


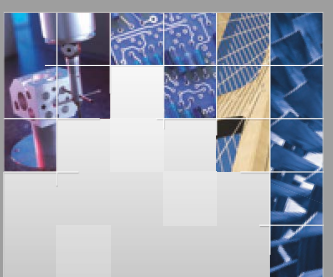

\section{Enfincering}
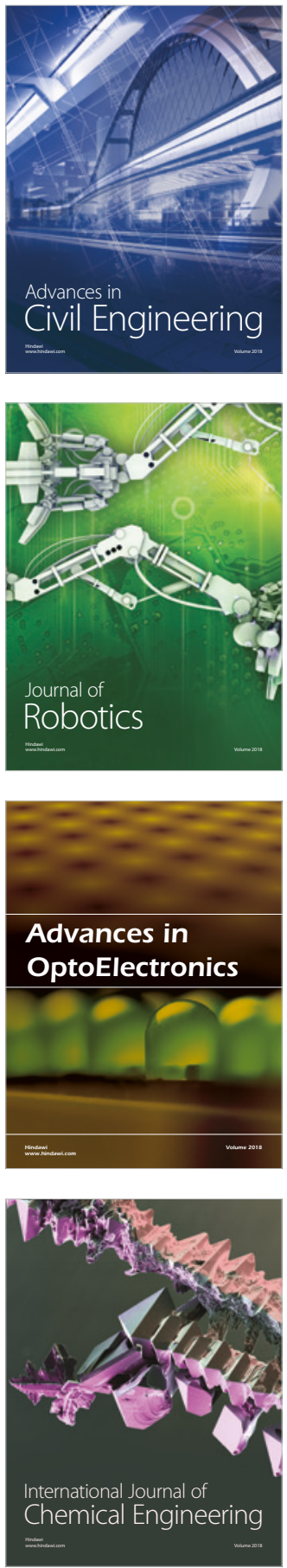

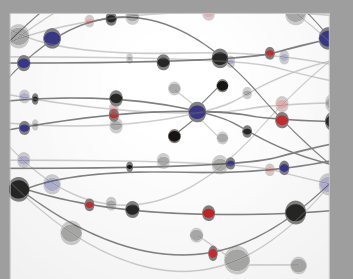

\section{Rotating \\ Machinery}

The Scientific World Journal

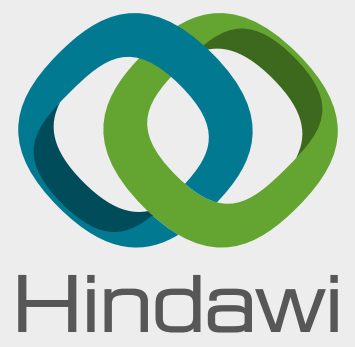

Submit your manuscripts at

www.hindawi.com
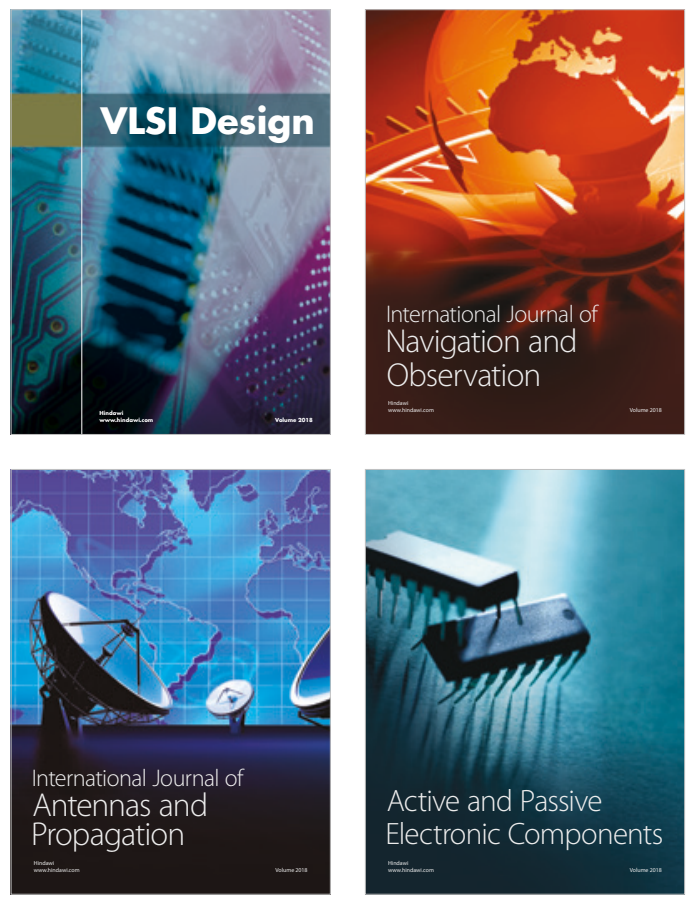
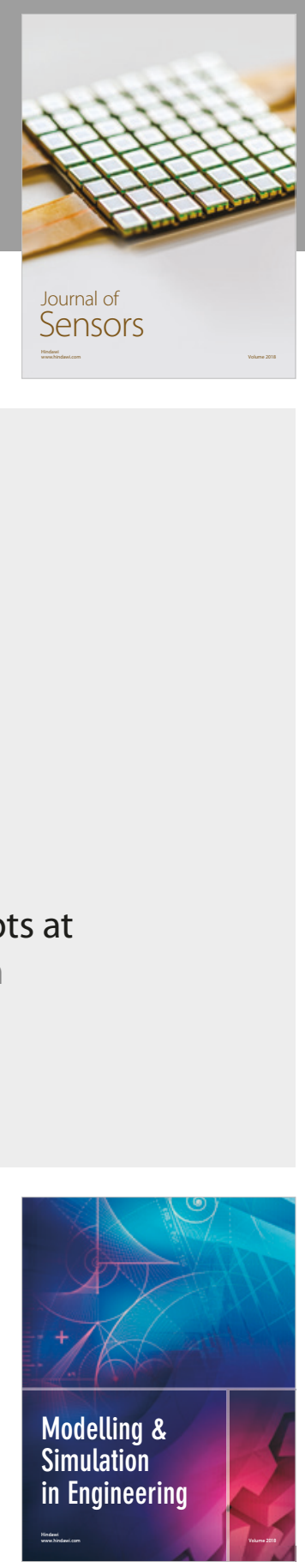

\section{Advances \\ Multimedia}
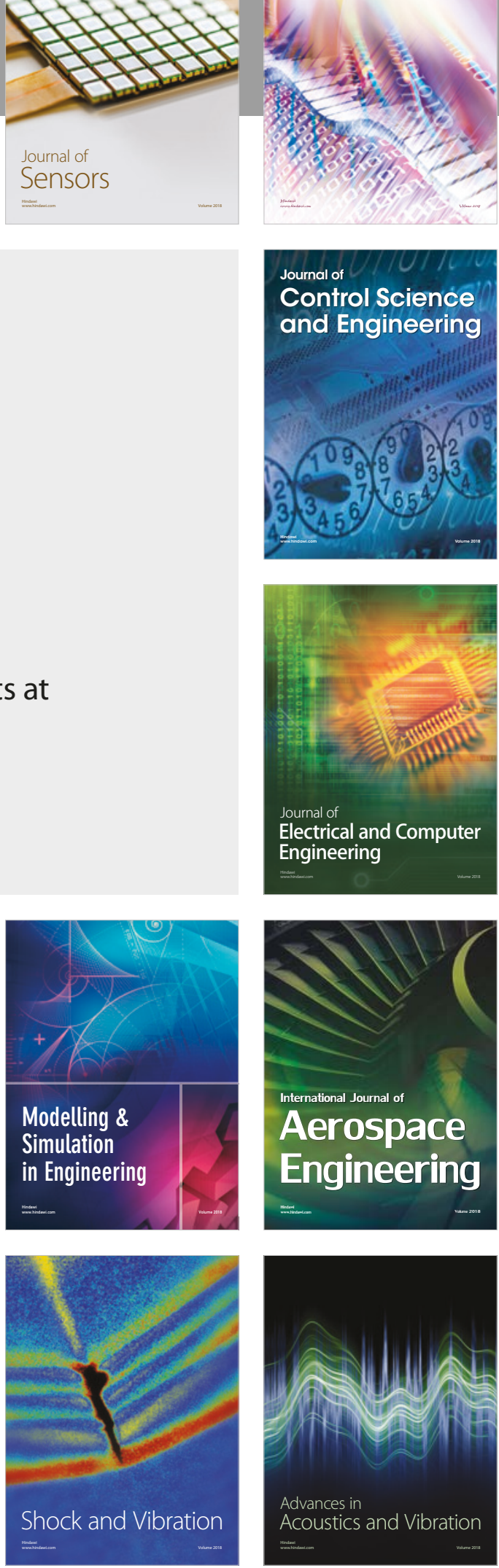